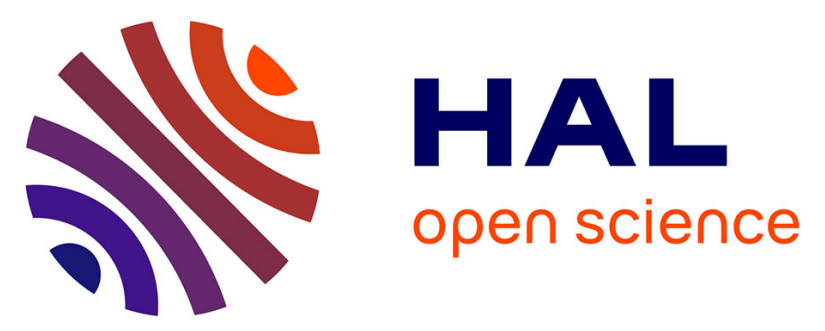

\title{
In Search of the Quantum-Electronic Origin of Color Change: Elucidation of the Subtle Effects of Alloying with Copper on $\approx 1.8 \mathrm{~nm}$ Gold Nanoclusters
}

Rajarshi Sinha-Roy, Xóchitl López-Lozano, R. Whetten, Pablo

García-González, H.-Ch. Weissker

\section{To cite this version:}

Rajarshi Sinha-Roy, Xóchitl López-Lozano, R. Whetten, Pablo García-González, H.-Ch. Weissker. In Search of the Quantum-Electronic Origin of Color Change: Elucidation of the Subtle Effects of Alloying with Copper on $\approx 1.8 \mathrm{~nm}$ Gold Nanoclusters. Journal of Physical Chemistry C, 2017, 121 (10), pp.5753 - 5760. 10.1021/acs.jpcc.7b00581 . hal-01720730

\author{
HAL Id: hal-01720730 \\ https://hal.science/hal-01720730
}

Submitted on 7 May 2018

HAL is a multi-disciplinary open access archive for the deposit and dissemination of scientific research documents, whether they are published or not. The documents may come from teaching and research institutions in France or abroad, or from public or private research centers.
L'archive ouverte pluridisciplinaire HAL, est destinée au dépôt et à la diffusion de documents scientifiques de niveau recherche, publiés ou non, émanant des établissements d'enseignement et de recherche français ou étrangers, des laboratoires publics ou privés. 


\section{In Search of the Quantum-Electronic Origin of}

\section{Color Change: Elucidation of the Subtle Effects of Alloying with Copper on $\approx 1.8 \mathrm{~nm}$ Gold}

\section{Nanoclusters}

Rajarshi Sinha Roy, ${ }^{\dagger, \ddagger}$ Xóchitl López Lozano, R.L. Whetten, Pablo García González, ${ }^{\S, \ddagger}$ and H.-Ch. Weissker ${ }^{*, \dagger, \uparrow}$

Aix Marseille University, CNRS, CINaM UMR 7325, 13288, Marseille, France, European Theoretical Spectroscopy Facility, Department of Physics \& Astronomy, The University of Texas at San Antonio, One UTSA circle, 78249-0697 San Antonio, TX., USA, and Departamento de Física Teórica de la Materia Condensada and Condensed Matter Physics Center (IFIMAC), Universidad Autónoma de Madrid, E-28049 Cantoblanco, Madrid, Spain

E-mail: weissker@cinam.univ-mrs.fr

${ }^{*}$ To whom correspondence should be addressed

${ }^{\dagger}$ CINAM

${ }^{\ddagger} \mathrm{ETSF}$

IUTSA

$\S$ UAM 


\begin{abstract}
Experiments on the $\mathrm{Au}_{144}(\mathrm{SR})_{60}$ class of cluster compounds have demonstrated the emergence of an absorption peak at $550 \mathrm{~nm}(2.25 \mathrm{eV})$ upon adding copper to the pure $\mathrm{Au}$ samples; in some cases, as little as one $\mathrm{Cu}$ atom seems to have this effect [Dharmaratne et al., Chem. Commun. 2014, 50, 1722-1724, and Bhattarai et al., J. Phys. Chem. C 2015, 119, 10935-10942]. These results are highly surprising as they appear to indicate that the combination of two metals that are not plasmonic in this size range produces a plasmon-like resonance.

Based on calculations using time-dependent density-functional theory (TDDFT), Malola et al. have recently concluded that "Copper Induces a Core Plasmon in Intermetallic $A u_{(144,145)-x} C u_{x}(S R)_{60}$ Nanoclusters." [J. Phys. Chem. Lett. 2015, 6, 515520]. In the present letter we show that these conclusions are erroneous; systematic TDDFT calculations on both bare and ligand-covered clusters do not show the emergence of any clear resonance, plasmonic or other. Instead, suppression of intensity in the range of interest between 2.0 and $3.5 \mathrm{eV}(620$ to $350 \mathrm{~nm})$ and small red shifts of weak spectral features may indeed change the color of the samples and may be seen as inducing a broad bump in the spectra. However, no clear increase in intensity if observed at the energy of interest $(2.25 \mathrm{eV} \approx 550 \mathrm{~nm})$ that would indicate the emergence of a plasmonic resonance. Other hypotheses will have to be explored to explain the experimental results which show a strong resonance developing.
\end{abstract}

\title{
Keywords
}

TDDFT, ab initio, bi-metallic, $\mathrm{AuCu}$, nanoalloy, surface-plasmon resonance, plasmon emergence, monolayer-protected 


\section{Introduction}

Noble-metal nanoclusters play an immensely important role as critical agents in a number of emerging technologies - in optics; in electronics; in diagnostic and therapeutic medicine. ${ }^{1-6}$ They can act as intense pigments of high durability in spectral ranges uniquely adapted to human color-vision. The localized surface-plasmon resonance (LSPR), which for many clusters dominates the visible (VIS) spectral region with a broad and smooth peak, ${ }^{7,8}$ is the physical mechanism exploited in most of the aforementioned applications. Nonetheless, while the general aspects of the clusters' optical response are well understood, a full understanding of the precise mechanisms governing the emergence of plasmon resonances and their coupling other elementary excitations is still missing. In particular, the transition from small moleculelike clusters, with their discrete electronic states and spectra, to larger metallic and plasmonic nanoparticles reflects the quantum nature of the clusters. ${ }^{9-11}$ A large number of studies have focused on different aspects of this transition: On the one hand, the emergence of the LSPR has been investigated for gold clusters, where it seems to take place between 150 and 330 atoms, ${ }^{12,13}$ with smaller Au clusters not showing the resonances. The presence of the plasmon depends on the underlying electron-hole transition spectrum which could lead either to fragmentation (if the e-h transitions are of s-p character) or suppression (if they are of d character). The exact conditions (geometry, chemical composition and configuration, and environment including presence and nature of ligand layers) are not entirely understood. On the other hand, the information content of the optical spectra provides precious information concerning the discrete electronic levels in the small clusters and on the way they develop into bands for bigger particles. ${ }^{14}$ In addition to the large number of gold clusters, ligand-stabilized silver clusters have likewise been synthesized. ${ }^{15,16}$

The thiolate ligand-protected $\mathrm{Au}_{144}(\mathrm{SR})_{60}$ cluster compound is an exceptionally well suited benchmark system for the investigation of optical properties in this transition sizerange, and this for a number of reasons. First, its diameter $(D \approx 1.8 \mathrm{~nm})$ lies in the size range where the LSPR starts to develop in Au clusters, but also where the transition from 
molecular-cluster to metallic-nanoparticle behaviors occurs. It shows itself no strong resonance in the visible spectra. ${ }^{12-14}$ Second, the cluster happens to be highly stable and has been studied by a number of different research groups. ${ }^{14,17-20}$ Third, the cluster compound exhibits icosahedral symmetry ${ }^{21,22}$ which implies a high degree of degeneracy of its electronic

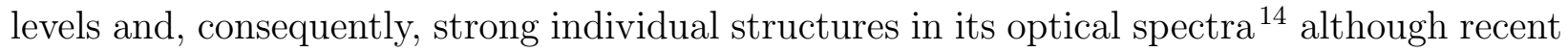
studies indicate polymorphism in certain samples. ${ }^{23,24}$ Finally, it has been shown experimentally that the precise nature of the ligand rest group (denoted $\mathrm{R}$ ) bears little influence on the optical spectra. For a collection of results see Ref. 25.

Among the three coinage metals, silver is optically different from $\mathrm{Au}$ and $\mathrm{Cu}$ because the onset of interband transitions from the $d$ band occurs at about $4 \mathrm{eV}$ in silver, whereas in $\mathrm{Au}$ and $\mathrm{Cu}$, it occurs at $\approx 2 \mathrm{eV}$. Therefore, silver behaves more like a free-electron metal and shows a clear LSPR down to very small sizes, ${ }^{26}$ although this depends also on the cluster surface; ligand-protected clusters of fewer than $\approx 150$ silver atoms seem to loose the plasmonic character. ${ }^{16}$ By contrast, in $\mathrm{Au}$ and $\mathrm{Cu}$, interband transitions involving $d$ electrons couple strongly with the LSPR, as much so as to make it disappear for small clusters, thereby creating the above-mentioned phenomenon of plasmon emergence/disappearance in dependence on cluster size. ${ }^{12,13,27}$ In view of these differences, it is natural to look at nanoalloys of these materials. Mixing of gold with silver has been studied, both experimentally and theoretically, for both bare ${ }^{27,28}$ and ligand-covered ${ }^{29,30}$ clusters, showing in general a smooth change of the spectra with changing composition, whereby the outermost layer of bare clusters strongly influences the properties. ${ }^{28}$ Insertion of a $\mathrm{Cu}$ core into a small silver cluster has been shown to strongly suppress the LSPR. ${ }^{31}$

Recent experiments have attempted to alloy gold and copper in the $\mathrm{Au}_{144}(\mathrm{SR})_{60}$ class of cluster compounds, leading to the most extraordinary results: while both $\mathrm{Au}$ and $\mathrm{Cu}$ at this size do not show a plasmonic resonance, insertion of copper into the gold cluster samples leads to the development of a peak at $550 \mathrm{~nm}(2.25 \mathrm{eV})$, reminiscent of the LSPR. ${ }^{32,33}$ This outstanding result — the combination of two non-plasmonic metals (at this size) apparently 
leading to the development of a plasmon resonance - calls out for explanation. The result is even more surprising in that it indicated that in some cases, a single copper atom might be sufficient to induce this effect, ${ }^{33}$ although this was not the case in the experiments performed by the Dass group. ${ }^{32}$ In order to understand this highly unexpected behavior, Malola et al. have carried out time-dependent density-functional theory (TDDFT) calculations and concluded that "Copper Induces a Core Plasmon in Intermetallic $A u_{(144,145)-x} C u_{x}(S R)_{60}$ Nanoclusters." 34 Substituting copper for gold atoms in a number of different configurations and compositions, these authors obtain the development of a peak at about $550 \mathrm{~nm}$ compared to the spectrum of the pure $\mathrm{Au}_{144}(\mathrm{SR})_{60}$, which is interpreted as a plasmonic resonance. Even the insertion of one copper atom in the center vacancy of the pure $\operatorname{Au}_{144}(\mathrm{SR})_{60}$ has been interpreted to have this effect.

In the present letter, we show that these conclusions are erroneous. The introduction of copper into the gold clusters leads essentially to the suppression of intensity at some wavelengths as well as to small red shifts in energy of some spectral features. The combination of these rather weak effects leads then in some case to spectra that exhibit a weak hump at the wavelength in question, at around $550 \mathrm{~nm}(\approx 2.2 \mathrm{eV})$. However, no indication is found of any increase of intensity in the spectra compared to those of the pure Au clusters, as it would correspond to a plasmonic resonance developing. We note that the energy range in question corresponds exactly to the onset of interband transitions from the d electrons $(2.0 \ldots 2.5 \mathrm{eV})$ and to the region where the LSPR in pure gold clusters should emerge. ${ }^{27}$ In other words, in this energy range there is a strong coupling between d-electron excitations and the nascent surface plasmon. Therefore, any statement about the precise nature of spectral features has to be made with extreme care.

The results depend only very slightly on the details of the structural model, the kind of ligand rest group used in the calculations, and on the approximations used in the calculations. This allows for the strong general conclusion that alloying with copper does not create plasmonic resonances in gold clusters in this size range, i.e., around $1.8 \mathrm{~nm}$, where the spectra 

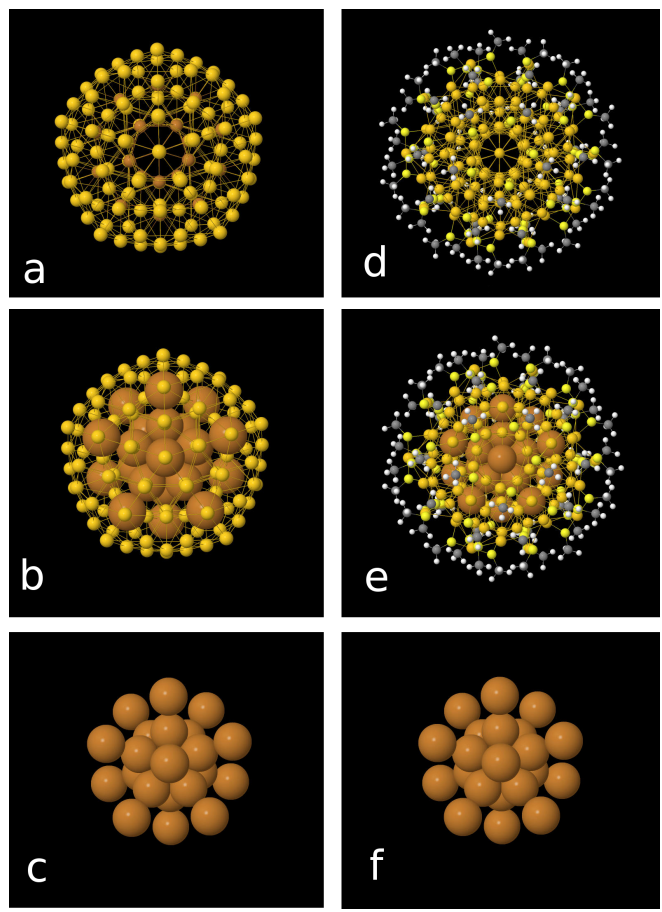

Figure 1: Structure of the bare 147-atom clusters (left-hand side, panels a-c) and the ligandprotected $\mathrm{Au}_{144}(\mathrm{SR})_{60}$ clusters (right-hand side, d-f). Uppermost panels: complete pure-Au clusters; middle panels: alloying with 25 copper atoms, which leads to $\mathrm{Au}_{122} \mathrm{Cu}_{25}$ for the bare and to $\mathrm{Au}_{120} \mathrm{Cu}_{25}(\mathrm{SR})_{60}$ for the ligand-protected cluster. In the ligand-protected structure, the central vacancy of the pure-gold structure is filled with a copper atom. The copper atoms are represented with a larger radius for the sake of visibility. Lower panels $(\mathrm{c}, \mathrm{f})$ : copper atoms only ( 25 in both cases). The distribution of the copper atoms is identical in the bare and the ligand-protected clusters and corresponds to "model 9" of Ref. 34.

do not yet show any clear LSPR. Regrettably, this means that the exciting experiments cannot be explained in this way; different mechanisms will have to be considered for their explanation.

\section{Absorption spectra}

As sketched in Fig. 1, we study the inclusion of $\mathrm{Cu}$ into $\mathrm{Au}$ clusters by replacing individual gold atoms by copper atoms (and, in some cases, filling the central vacancy, which leads to numbers of atoms of 144 or 145 for the $\mathrm{Au}_{144}$-derived liganded clusters, and an unchanged 147 for the bare clusters). We use the time-evolution formalism ${ }^{35}$ of time-dependent density- 


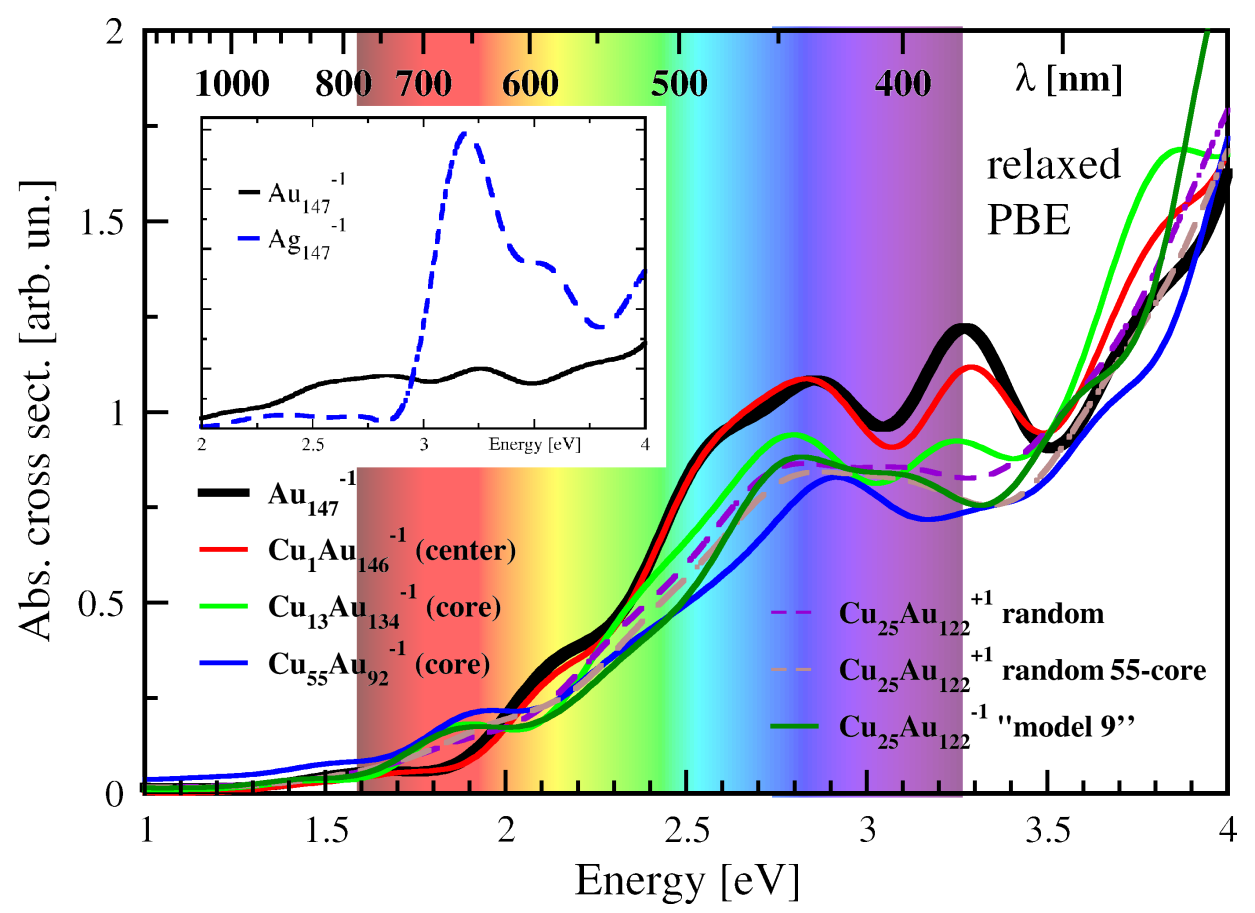

Figure 2: Bare clusters: Absorption spectrum of the bare 147-atom Au icosahedral cluster (thick black line) compared to different Au-Cu alloy clusters, all relaxed using the PBE GGA functional (spectra for LDA-relaxed clusters are shown in Figure S1 of the SI). We show the spectra corresponding to: insertion of one $\mathrm{Cu}$ atom in the center vacancy $\left(\mathrm{Cu}_{1} \mathrm{Au}_{146}\right.$, red), the replacement of the positions of the 13-atom core by $\mathrm{Cu}$ atoms $\left(\mathrm{Cu}_{13} \mathrm{Au}_{134}\right.$, green), the same for the 55-atom core $\left(\mathrm{Cu}_{55} \mathrm{Au}_{92}\right.$, blue), and $\mathrm{Cu}_{25} \mathrm{Au}_{122}$ clusters with random configurations: $25 \mathrm{Cu}$ atoms randomly distributed throughout the cluster (violet) and throughout the 55atom core (brown). Finally, the dark green line corresponds to model \# 9 of Reference ${ }^{34}$ (see section "Technical Details"). In the inset, a comparison of the absorption spectra of icosahedral $\mathrm{Au}_{147}$ and the plasmonic $\mathrm{Ag}_{147}$ is shown. Clearly, no resonance emerges in any of the considered $\mathrm{Au}-\mathrm{Cu}$ clusters, there is rather a suppression of intensity throughout the spectral range of interest ( $\omega \sim 2.0-3.5 \mathrm{eV}$, i.e., 620 to $350 \mathrm{~nm})$, along with a small red-shift of the shoulder at $2.1 \mathrm{eV}$ in pure gold to about $1.9 \mathrm{eV}$ for the gold-copper alloy clusters. For the sake of visibility, we present here spectra from a time evolution of $15 \mathrm{fs}$, corresponding roughly to a broadening of $0.3 \mathrm{eV}$ in the spectra. To highlight the rich details in the spectral features, we show spectra with much higher spectral resolution $(0.18 \mathrm{eV}$ and $0.018 \mathrm{eV})$ in the Supporting Material, Figures S2 and S3.

functional theory and employ the real-space pseudopotential code octopus. ${ }^{36}$ The technical details are described in the Technical Details section. We have demonstrated the applicability of this approach to bare ${ }^{31}$ and ligand-protected clusters ${ }^{14,25}$ as well as to nanoalloys. ${ }^{28}$ The results are in agreement with those obtained using the Casida approach (LR-TDDFT), ${ }^{37,38}$ which is illustrated in the Supplementary Material of Ref. 14 for the thiolate-ligand covered 
$\mathrm{Au}_{38}$ cluster and, for a bare Au cluster, in the Supplementary Material of Ref. 39.

Bare icosahedral 147-atom clusters: Before we discuss the $\mathrm{Au}_{144}(\mathrm{SR})_{60}$ compound, we study the far simpler case of bare 147-atom icosahedral clusters. These clusters of $\approx 1.6 \mathrm{~nm}$ diameter have roughly the same number of gold atoms as the $\mathrm{Au}_{144}(\mathrm{SR})_{60}$ and exhibit likewise icosahedral symmetry. Unlike in the $\mathrm{Au}_{144}(\mathrm{SR})_{60}$ cluster, the central vacancy is filled. They show very clearly the difference between silver with a very strong LSPR visible in the spectra and gold without. ${ }^{31}$ In fact, the 147-atom Au clusters appear to be just below the size where the LSPR emerges, which happens between $\approx 150$ and 330 atoms. ${ }^{12,13}$ The calculated spectrum of $\mathrm{Au}_{147}$ is shown in Fig. 2 where the spectrum of the corresponding silver cluster with its strong resonance is shown in the inset for comparison.

Also in Fig. 2, we show the comparison of spectra following the incorporation of one, 25, and $55 \mathrm{Cu}$ atoms in the cluster. The inclusion of one $\mathrm{Cu}$ atom replacing the central $\mathrm{Au}$ atom leads to minimal changes in the spectra. Adding more $\mathrm{Cu}$, whereby we keep the cluster symmetric except in the case of random distributions where the symmetry is obviously broken, we obtain a clear and coherent picture: the changes upon adding copper are such that the structures between 2.0 and $3.5 \mathrm{eV}(620$ to $350 \mathrm{~nm})$ are smoothened and that the spectral intensity is reduced at some energies. The shoulder at $2.2 \mathrm{eV}$ is red-shifted to about $1.9 \mathrm{eV}$ $(650 \mathrm{~nm})$. No indication of plasmon emergence is found. This is likewise true for clusters relaxed using the local density approximation (LDA) to the exchange-correlation functional instead of the generalized-gradient approximation (GGA). These spectra are shown in supplementary figure S1.

The present spectra are calculated with an evolution time of $15 \mathrm{fs}$, corresponding to a broadening of about $0.3 \mathrm{eV}$ in the spectra. For more finely resolved spectra, and in order to check for the development of narrow, weaker resonances that might go unnoticed in the calculation with this broadening, we show in Figure S2 of the Supplementary Material spectra calculated for longer evolution time, viz., 25 fs corresponding to $0.18 \mathrm{eV}$. In these more finely resolved spectra, the general findings are naturally the same. Comparing the copper-alloyed 
clusters with the pure-Au cluster, there is the clear suppression of intensity between 2.0 and $3.5 \mathrm{eV}$ as well as a red shift of the small peak at $2.15 \mathrm{eV}$ to $1.9 \mathrm{eV}$, which corresponds to the shoulders in Fig. 2. The findings are similar for all chemical configurations of the copper alloying.

Finally, the spectra with an extremely small broadening (250 fs evolution time corresponding roughly to $0.018 \mathrm{eV}$ broadening) in the supplementary figure S3 show that in the interesting range between 1.5 and $2.5 \mathrm{eV}$, there is some rearrangement of spectral intensity. However, no intensity increase is observed that would be characteristic of the emergence of a plasmonic resonance. This is particularly clear in view of the direct comparison, also in Figure S3, with the strong resonance exhibited by the equivalent silver cluster $\mathrm{Ag}_{147}$ with it's clear plasmonic resonance.

Ligand-covered 144-atom clusters: For the ligand-protected clusters, a number of additional parameters complicate the description of the system. Besides the structural relaxations in the available literature using either LDA or GGA, ${ }^{12,14,30}$ which leads to small but relevant changes ${ }^{25}$ the description of the ligand rest groups plays an important role, although the optical measurements are rather insensitive to the precise nature of the rest group $\mathrm{R}$ of the thiolate ligands (see reference 25 for a direct comparison of measurements). The TDDFT calculations need to reduce the number of atoms as far as possible due to numerical limitations. We have shown previously that the reduction of the ligand rest group to only $\mathrm{R}=\mathrm{H}$ is too drastic ${ }^{25}$ and kept the methyl group in our calculations, $\mathrm{R}=\mathrm{CH}_{3} \cdot{ }^{14,25}$ Another subtle but relevant factor is the symmetry of the system. The original model as published by López-Acevedo et al. ${ }^{40}$ has the "staple motives" mutually oriented in a way that does not respect the overall icosahedral symmetry of the compound. Following the experimental work of Wong et al. ${ }^{21}$ which indicated that all ligands are positioned in symmetry-equivalent positions, the model was refined so as to have the full icosahedral symmetry. ${ }^{22}$ The resulting small differences in the spectra of the respective structures are discussed in detail in a previous work. ${ }^{25}$ The study of Malola et al. ${ }^{34}$ uses the original, slightly less symmetric geometry, 

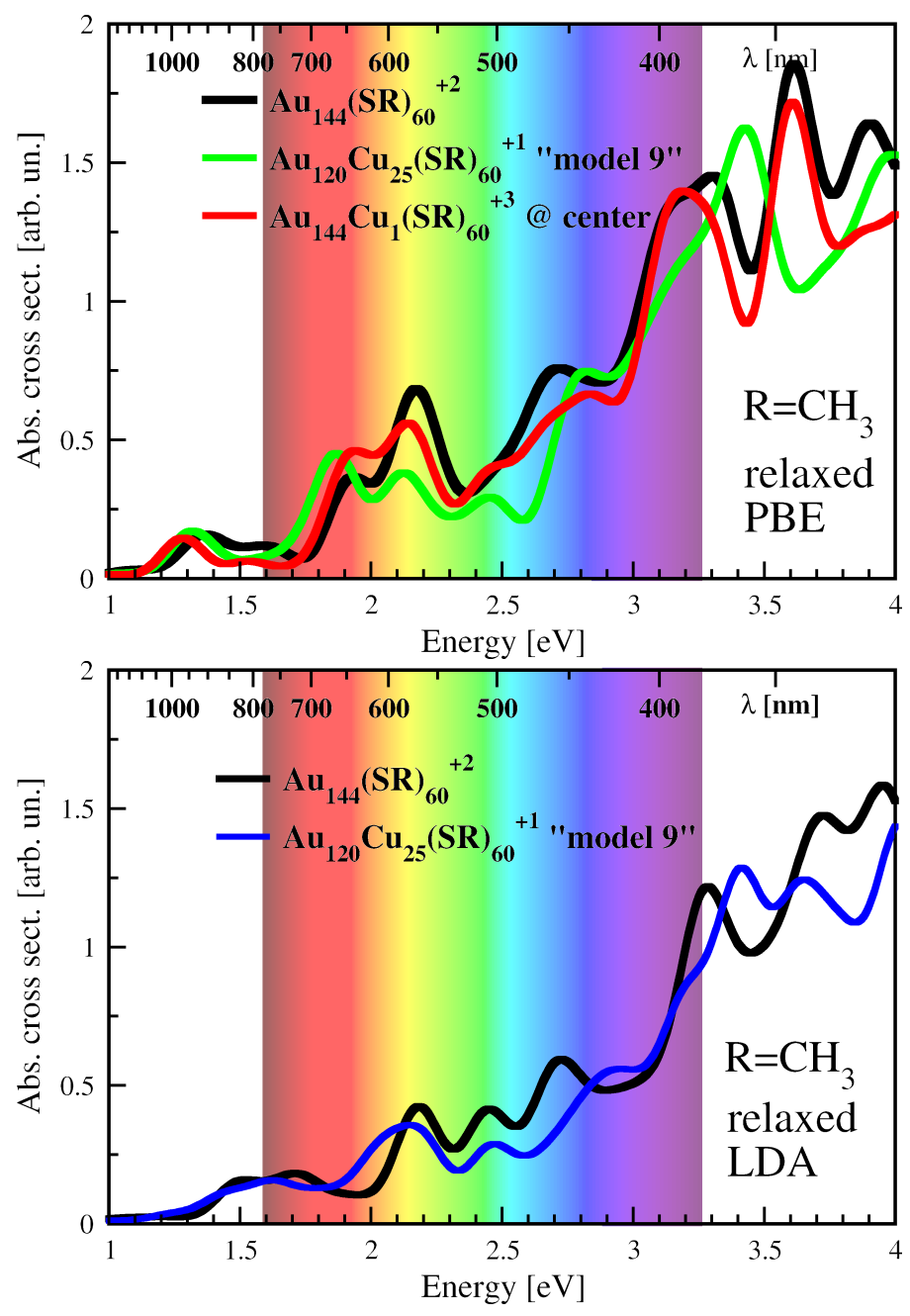

Figure 3: Monolayer-protected $\mathbf{A u}_{144}(\mathbf{S R})_{60}$ clusters: Change of the absorption spectra of $\mathrm{Au}_{144}(\mathrm{SR})_{60}$ upon alloying with copper. We compare the spectra of the pure-gold cluster (black) to those of the alloy clusters. "Model \#9" corresponds to the configuration of the same denomination used in reference 34 and is shown in the present Figure 1 . We use the rest group $\mathrm{R}=\mathrm{CH}_{3}$ and relaxation using the PBE (upper panel) and the LDA (lower panel) functionals. Spectra for $\mathrm{R}=\mathrm{H}$ are shown in the Supplementary Material, Figure $\mathrm{S} 4$. The insertion of one copper atom into the central vacancy has a small but noticeable effect. Upon adding 25 copper atoms, a suppression of intensity in the range between 2 and $3 \mathrm{eV}$ leads to a bump at around the desired energy, but there is clearly no increase of intensity that would be indicative of the emergence of a plasmonic resonance. These changes may modify the color of the samples, but they do not describe the emergence of a plasmonic resonance, i.e., the strong effects reported in the experimental studies. ${ }^{32,33}$ 
the minimal ligand rest group $\mathrm{R}=\mathrm{H}$, and ground-state relaxation with the LDA functional. The latter is motivated by the fact that for gold, the interatomic distances are better reproduced by LDA than when a GGA is used. However, naturally the presence of the ligands as well as of the $\mathrm{Cu}$ reduces the plausibility of this choice somewhat. Other previous studies have used the GGA function PBE for relaxation. ${ }^{14,25,30}$ It seems that the spectra using the fully symmetric structure, $\mathrm{R}=\mathrm{CH}_{3}$, and relaxation with $\mathrm{PBE}$ are the ones that are closest to experiment, compared to the spectra using different choices. ${ }^{12,14,25,30}$

In order to obtain generally valid results and to exclude the possibility that any of these parameters invalidate our conclusions, we treat all four different cases: ligand rest groups $\mathrm{R}=\mathrm{H}$ and $\mathrm{R}=\mathrm{CH}_{3}$ for, in both cases, structural relaxations using either LDA or GGA. Our results are shown in Fig. 3 for $\mathrm{R}=\mathrm{CH}_{3}$. Clearly, the addition of copper does not induce any strong increase of spectral intensity in the region of interest around $2.2 \mathrm{eV}$. Apart from a very small red-shift of the shoulder at about $2 \mathrm{eV}$, the changes due to the insertion of copper occur by suppression of spectral intensity. The use of GGA and LDA in the relaxation leads to slightly different spectra, as shown before for the pure $\mathrm{Au}_{144}(\mathrm{SR})_{60},{ }^{25}$ but the conclusions concerning the effect of alloying with copper do not differ between the two cases shown in Fig. 3.

Clearly, the choice of the methyl group $\mathrm{CH}_{3}$ is physically better motivated than the reduction of the ligand rest group to just one hydrogen atom. However, in order to doublecheck for the possibility that the choice of the rest group influences the conclusions, we show the spectra for $\mathrm{R}=\mathrm{H}$, which we obtain by replacing each methyl group by an $\mathrm{H}$ atom and relaxing, in the lower panels of Supplementary Fig. S4. The situation for the PBE-relaxed clusters is as clear as for the calculations using $\mathrm{CH}_{3}$, there is again a small red-shift upon insertion of copper but no emergence of any resonance.

The case of the LDA-relaxed $\mathrm{R}=\mathrm{H}$ calculation is slightly more intricate. It is interesting to note that in this case, for the pure $\mathrm{Au}_{144}(\mathrm{SR})_{60}$, the LDA-relaxed less symmetric structure with $\mathrm{R}=\mathrm{H}$ leads to a somehow more "flat", rounded structure between 1.9 and $2.7 \mathrm{eV}$ 
compared to the fully symmetric structure. Only in this case, insertion of copper produces indeed an - albeit very weak - increase of intensity in a very narrow range around the desired energy of $2.2 \mathrm{eV}$. Surprisingly, this is already the case when one single $\mathrm{Cu}$ atom is placed in the central vacancy. However, the change is small and the intensity of this peak does hereafter not change when the copper content is increased to 25 atoms. There is, instead, rather a suppression of intensity between 2.5 and $3.0 \mathrm{eV}$, which contributes to the impression of a peak developing at $2.2 \mathrm{eV}$. Nonetheless, comparing the respective spectra directly and to scale (comparing, in particular, the calculation using the physically preferable $\mathrm{CH}_{3}$ rest group) it is clear that the interplay of the subtle structural changes leading to a fortuitous rearrangement of oscillator strength is at the origin of the development of the little peak; no emergence of any resonance is seen.

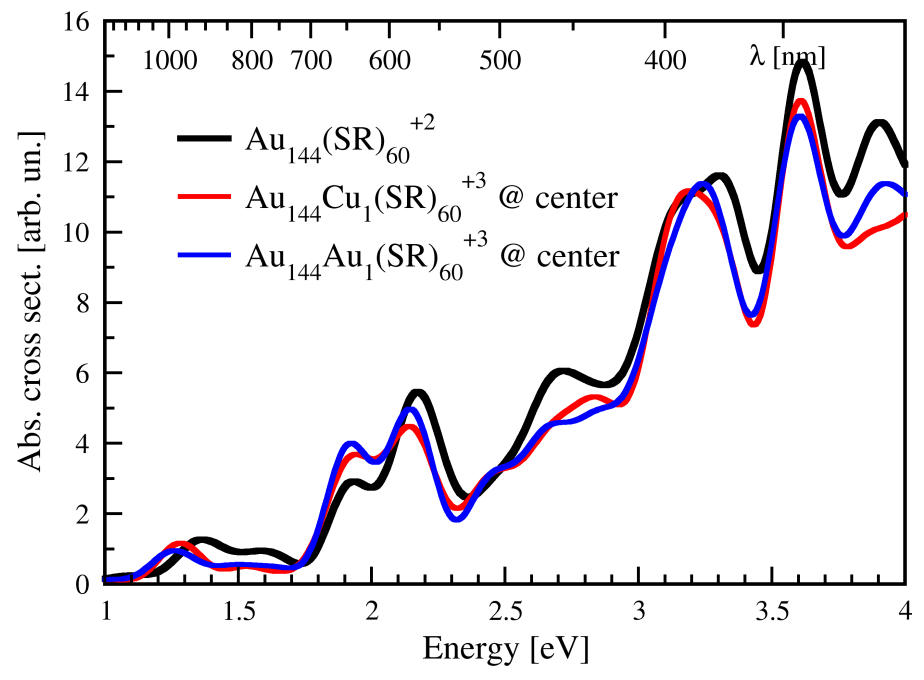

Figure 4: One copper atom: chemical vs. geometrical effect: Effect of the introduction of either a copper or a gold atom into the central vacancy of the pure $\mathrm{Au}_{144}(\mathrm{SR})_{60}$ cluster compound in order to distinguish the purely geometric effect and the chemical effect. Ground state relaxation done using PBE, rest group $\mathrm{R}=\mathrm{CH}_{3}$. The two spectra deviate little but noticeably from the pure gold spectra but are almost identical; the entire effect is, consequently, geometric.

One copper atom: It is interesting to note that even the addition of one copper atom in the central vacancy leads to noticeable changes in the spectrum, although in general, they remain small (where the case of the LDA-relaxed structures with $\mathrm{R}=\mathrm{H}$ is somewhat 
an exception.) We have demonstrated in the previous paragraph that subtle changes in the geometry are responsible for the deceptive impression that a LSPR-like resonance might develop. In order to distinguish between the geometrical effect and the chemical effect of inserting a copper atom, we compare, in Fig. 4, the effect of adding a copper atom and that of adding a gold atom in the central vacancy. The addition of the gold atom allows for the consideration of only the effect of a geometric change, whereas the addition of $\mathrm{Cu}$ induces both a geometric and a chemical change. The finding in Fig. 4 is that the changes caused by the introduction of either a gold atom or a copper atom are similar to a very high degree. This allows for the important conclusion that the effect is almost entirely structural, with little influence of the chemical nature of the central atom. Again, this indicates that $\mathrm{Cu}$ does not induce a plasmonic resonance.

\section{Dynamical polarizability and induced density}

As it is well known, in the dipole approximation the absorption cross section corresponding to an external electromagnetic (EM) plane wave of frequency $\omega$ whose $E$-field is polarized along the $z$ direction [that is, $\mathbf{E}_{\text {ext }}(t)=E_{0} \cos (\omega t) \mathbf{e}_{z}$ ] is given by

$$
\sigma_{\mathrm{abs}}(\omega)=\frac{4 \pi \omega}{c} \Im \alpha(\omega)
$$

where $c$ is the speed of light and $\alpha(\omega)$ is the dynamical polarizability. The latter is defined as the induced electric dipole divided by the amplitude $E_{0}$ of the incident electric field:

$$
\alpha(\omega)=-\frac{1}{E_{0}} \int z \delta n(\mathbf{r}, \omega) d \mathbf{r},
$$

$\delta n(\mathbf{r}, \omega)$ being the induced electron density. Note that according to the standard complex representation of harmonic functions, the time-dependent induced electron density is given 

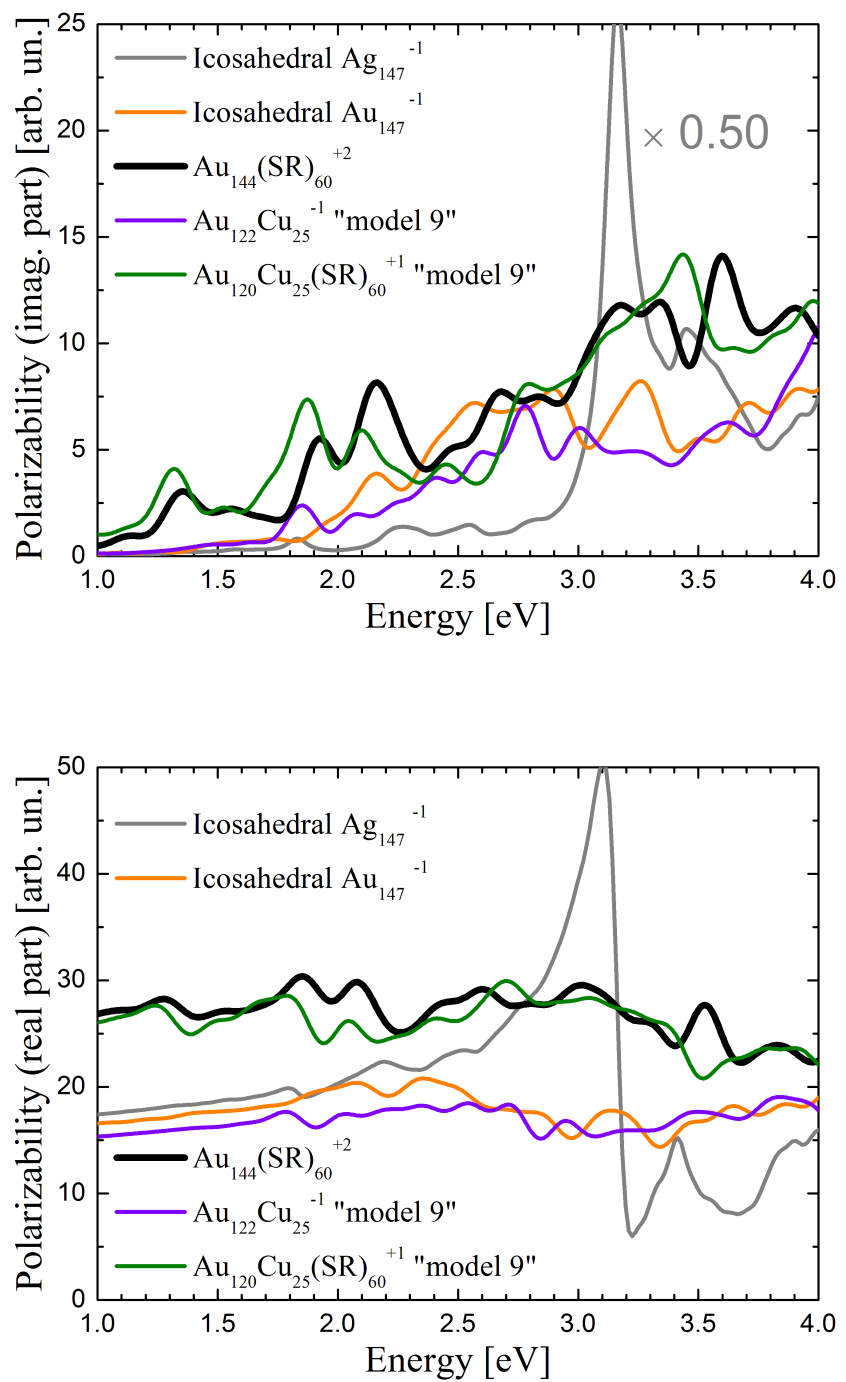

Figure 5: Dynamical polarizability: Real (bottom panel) and imaginary (top panel) parts of the dynamical polarizability, $\alpha(\omega)$, for selected bare and ligand-protected clusters. Thick gray line: bare $\mathrm{Ag}_{147}$; thick black line: bare $\mathrm{Au}_{147}$; green line: bare $\mathrm{Cu}_{25} \mathrm{Au}_{122}$ according to "model \#9" of Reference 34 (cf., the present Figure 1); blue line: ligand-protected $\mathrm{Au}_{144}(\mathrm{SR})_{60}$ cluster; red line: ligand-protected $\mathrm{Cu}_{25} \mathrm{Au}_{120}(\mathrm{SR})_{60}$ cluster (again "model \#9"; rest group $\mathrm{R}=\mathrm{CH}_{3}$ ). While the real part of the polarizability for the plasmonic silver cluster $\operatorname{Ag}_{147}$ cluster exhibits a sharp variation at the LSPR frequency $\left(\omega_{\mathrm{P}} \simeq 3.15 \mathrm{eV}\right)$, there is no noticeable structure for the Au-based nanoparticles, regardless of the inclusion of $\mathrm{Cu}$ atoms. A broadening of $0.05 \mathrm{eV}$ has been used.

by

$$
\delta n(\mathbf{r}, t)=\cos (\omega t) \Re \delta n(\mathbf{r}, \omega)+\sin (\omega t) \Im \delta n(\mathbf{r}, \omega),
$$


and the real [imaginary] parts are then related to oscillations in phase [out of phase with a phase difference of a quarter of period] with the external E-field. Therefore, the absorption is proportional to the imaginary part of the polarizability $\alpha(\omega)$, which accounts for outof-phase oscillations of the electron density with respect to the external $E$-field. However, further insights concerning the interaction between a nanoparticle and an external EM field can be obtained by analyzing the real part of $\alpha(\omega)$, that is, the in-phase induced electric dipole.

In extended bulk systems, where electron-hole transitions form a continuum, the plasmon is a well-defined and distinct elementary excitation. ${ }^{41}$ By contrast, for systems with a finite number of electrons the concept of collective excitation and its corresponding distinction from electron-hole excitations is more vague. In fact, a LSPR might be seen as an electron-hole excitation that is highly renormalized by the electron-electron interaction. ${ }^{42}$ This excitation can be coupled to surrounding electron-hole intraband transitions, in such a way that the LSPR acquires an effective width through a "Landau fragmentation" mechanism. ${ }^{43}$ However, as we have mentioned before, the coupling of the LSPR with interband transitions leads to the practical disappearance of the plasmon resonance. Such couplings prevent us from identifying a single spectral peak as "the" LSPR in many cases. Then, the signature of the existence of a LSPR is the concentration of spectral weight around a given frequency $\omega_{\mathrm{P}}$ that, for sufficiently large systems, would be close to the classical-optics prediction. This is precisely the situation in bare and ligand-protected Au clusters and related nanoalloys: the region where the LSPR is expected to appear is already occupied by a multitude of electron-hole transitions.

The concentration of spectral weight around $\omega_{\mathrm{P}}$ can not only be observed in the imaginary part of the dynamical polarizability; the real part of $\alpha(\omega)$ around the frequency of an isolated, well defined resonance must exhibit a change of sign (from positive to negative). The presence of less-intense nearby transitions can hamper this change of sign but, in any case, a sharp variation of $\Re \alpha(\omega)$ with negative slope is a clear indication of spectral-weight 
concentration and, therefore, of the existence of a LSPR. As we may see in Fig. 5, this is indeed the case for the icosahedral $\operatorname{Ag}_{147}$ cluster (a more detailed presentation including the use of a lower broadening are shown in the Supplementary Material, Fig. S5.). However, there isn't any distinctive behavior in both the real and imaginary parts of the polarizability of bare and ligand-protected $\mathrm{Cu} / \mathrm{Au}$ alloys indicating a $\mathrm{Cu}$-induced concentration of spectral weight in the region $2.0-2.5 \mathrm{eV}$. Hence, the analysis of the real part of the dynamic polarizability confirms the findings based on the direct comparison of the different absorption spectra.

Density dynamics: We need to point out one more issue. The time-dependent polarization of the system is connected to the movement of the electrons and, therefore, the dynamics of the electron density. A localized surface-plasmon resonance can be considered a collective oscillation of the quasi-free electrons, with modifications due to the atomistic inhomogeneity and the presence of the $d$ electrons in the noble metals. ${ }^{44}$ However, it is not enough to find such a dipole mode in the dynamics of the density, as this can be present even in cases where no prominent resonance is present in the spectra. We show the induced density in the pure bare $\mathrm{Au}_{147}$ cluster after quasi-monochromatic laser excitation in Fig. S6 of the Supporting Material. Even in this case, where the spectrum clearly does not exhibit any strong plasmonic resonance, we find the sort of dipole pattern that is reminiscent of a plasmonic excitation, including the out-of-phase movement of the d electrons that shows the screening of the oscillation. In other words, the presence of a dipole mode in the electron density dynamics, even if reminiscent of an LSPR dipole mode, does not allow for clear conclusions about the plasmonic nature of spectral features. 


\section{Summary and conclusions}

Motivated by experimental findings and recently published calculations which concluded that "Copper Induces a Core Plasmon in Intermetallic A $u_{(144,145)-x} C u_{x}(S R)_{60}$ Nanoclusters.", ${ }^{34}$ we have calculated the changes of the optical response of both the bare 147-atom icosahedron and of the $\mathrm{Au}_{144}(\mathrm{SR})_{60}$ cluster compound upon alloying with copper. Our systematic results show that the conclusions of Ref. 34 are erroneous.

Copper atoms are replacing gold atoms of the pure cluster (and, in addition, are inserted into the central vacancy of $\left.\mathrm{Au}_{144}(\mathrm{SR})_{60}\right)$. Considering a number of different situations as far as the ground-state relaxation of the structures is concerned (LDA vs. PBE), the choice of the rest group of the thiolate ligands $\left(\mathrm{R}=\mathrm{H}\right.$ vs. $\mathrm{R}=\mathrm{CH}_{3}$ ), and the subtle differences in symmetry/geometry discussed previously, ${ }^{25}$ we show that the addition of copper does not induce the development of any prominent resonance, plasmonic or other. The main changes are small and consist mostly in a) suppression of spectral intensity in the range between 2.0 and $3.5 \mathrm{eV}$ (620 to $350 \mathrm{~nm})$, and b) a red-shift of the lowest spectral features. Moreover, we demonstrate that the small but noticeable changes upon insertion of a single copper atom in the central vacancy of the pure $\mathrm{Au}_{144}(\mathrm{SR})_{60}$ are almost entirely due to the geometric effect of the copper insertion.

These findings mean that the experiments in which the development of an LSPR-like peak has been observed cannot be explained by the simple hypothesis that the insertion of copper changes the optical response be inducing a plasmonic resonance. Other hypotheses will need to be explored in order to obtain a fundamental understanding of the effects at play in these experiments.

\section{Technical details}

The ionic ground-state relaxations have been done using the VASP $\operatorname{code}^{45-47}$ with the projector-augmented wave method (PAW) ${ }^{47}$ The force tolerance has been set to $0.01 \mathrm{eV} / \AA$. 
The absorption spectra are calculated in TDDFT using the real-space code octopus. ${ }^{36,48}$ Following a ground-state calculation, spectra are obtained with the time-evolution formalism $^{35}$ and the PBE-GGA exchange-correlation functional ${ }^{49}$ for all spectra shown, including those where the structural relaxation was done using LDA. Norm-conserving TroullierMartins pseudopotentials ${ }^{50}$ have been used which, similarly as in our previous work, ${ }^{14,25}$ include the $d$ electrons in the valence (11 valence electrons for each $\mathrm{Au}$ or $\mathrm{Cu}$ atom, i.e., $\sim 2,500$ active electrons). The spacing of the real-space grid was set to $0.20 \AA$ for the pure$\mathrm{Au}$ clusters, and to $0.16 \AA$ for the clusters containing copper. The radius of the spheres centered around each atom which make up the calculation domain was $5 \AA$. The clusters have been charged as indicated such that the degenerate HOMO states were fully occupied.

The time-evolution formalism is equivalent to the transition-based Casida formalism (LRTDDFT). ${ }^{37,38}$ A comparison of the time-evolution results with a transition-based calculation is shown in the Supplementary Material of Ref. 14 for the thiolate-ligand covered $\mathrm{Au}_{38}$ cluster. For a bare Au cluster, a similar comparison is shown in the supplementary material of Ref. 39. The good agreement shows likewise that the technical parameters of our calculations are well controlled.

Apart from the customary 147-atom icosahedron that has been studied in many works, we consider the following structural models of $\mathrm{Au}_{144}(\mathrm{SR})_{60}$ and its alloys with copper. (For a detailed analysis of the differences between these structures, refer to Ref. 25.)

- The fully symmetric, GGA-relaxed $\mathrm{Au}_{144}(\mathrm{SR})_{60}$ with $\mathrm{R}=\mathrm{CH}_{3}$ from the work of Bahena et al., Ref. 22, is used as starting structure. For the respective comparison, this structure has also been re-relaxed using LDA .

- The $\mathrm{Au}_{144}(\mathrm{SR})_{60}$ with $\mathrm{R}=\mathrm{CH}_{3}$ is obtained from the same structure by replacing the methyl group with $\mathrm{H}$ atoms and re-relaxing.

- For comparison (only shown in the Supporting Information) we have also used the "Malola et al. structure" as employed in the calculations of Ref. 12. The structure 
is derived from the original, less symmetrical López-Acevedo et al. structure $^{40}$ by reducing the rest group to $\mathrm{R}=\mathrm{H}$, probably resulting in a partial symmetrization as discussed in Ref. 25.

- Copper (or, in one case, gold) is introduced into the center vacancy (resulting in $\left.\mathrm{Au}_{144} \mathrm{Cu}_{1}(\mathrm{SR})_{60}\right)$. For the higher copper content, $\mathrm{Au}_{120} \mathrm{Cu}_{25}(\mathrm{SR})_{60}$, we use the model structure that is called model \#9 in Malola et al. which consists of a 13-atom Cu core (filled central vacancy) and $12 \mathrm{Cu}$ atoms distributed symmetrically in the 42 -atom shell around the 13 -atom core so as to conserve the icosahedral symmetry. ${ }^{34}$

\section{Acknowledgments}

Useful discussions with Antonio Fernández-Domíguez are gratefully acknowleded. This work has been carried out thanks to the support of the A*MIDEX grant ( ${ }^{\circ}$ ANR-11-IDEX-000102) funded by the French Government "Investissements d'Avenir" program. We acknowledge support from the French National Research Agency (Agence Nationale de Recherche, ANR) in the frame of the project "FIT SPRINGS," ANR-14-CE08-0009. XLL acknowledges funding from NSF-DMR-1103730, NSF-PREM DMR-0934218. RLW acknowledges the support of the Welch Foundation under grant AX-1857. PGG acknowledges financial support from the Spanish Ministry of Economy and Competitiveness through the "María de Maeztu" Programme for Units of Excellence in R\&D (MDM-2014-0377) and through the research grant MAT2014-53432-C5-5-R. The work received computational support from Computational System Biology Core, funded by the National Institute on Minority Health and Health Disparities (G12MD007591) from the National Institutes of Health, as well as the Texas Advanced Computing Center (TACC) at The University of Texas at Austin and HPC resources from GENCI-IDRIS (Grant 096829). 


\section{References}

(1) Love, J. C.; Estroff, L. A.; Kriebel, J. K.; Nuzzo, R. G.; Whitesides, G. M. SelfAssembled Monolayers of Thiolates on Metals as a Form of Nanotechnology. Chemical Reviews 2005, 105, 1103-1170.

(2) Prashant K. Jain, I. H. E.-S., Xiaohua Huang; El-Sayed, M. A. Noble Metals on the Nanoscale: Optical and Photothermal Properties and Some Applications in Imaging, Sensing, Biology, and Medicine. Accounts of Chemical Research 2008, 41, 1578-1586.

(3) Hainfeld, J. F.; Liu, W.; Halsey, C. M.; Freimuth, P.; Powell, R. D. Ni-NTA-Gold Clusters Target His-Tagged Proteins. Journal of Structural Biology 1999, 127, 185 198.

(4) Ackerson, C. J.; Powell, R. D.; Hainfeld, J. F. In Cryo-EM Part A Sample Preparation and Data Collection; Jensen, G. J., Ed.; Methods in Enzymology; Academic Press, 2010; Vol. 481; pp 195 - 230.

(5) Bowman, M.-C.; Ballard, T. E.; Ackerson, C. J.; Feldheim, D. L.; Margolis, D. M.; Melander, C. Inhibition of HIV Fusion with Multivalent Gold Nanoparticles. Journal of the American Chemical Society 2008, 130, 6896-6897.

(6) Bresee, J.; Maier, K. E.; Boncella, A. E.; Melander, C.; Feldheim, D. L. Growth Inhibition of Staphylococcus aureus by Mixed Monolayer Gold Nanoparticles. Small 2011, 7, 2027-2031.

(7) Faraday, M. Noble Metals on the Nanoscale: Optical and Photothermal Properties and Some Applications in Imaging, Sensing, Biology, and Medicine. Philosophical Transactions: Royal Society London 1857, 147, 145-181.

(8) Craig F. Bohren, D. R. H. Absorption and Scattering of Light by Small Particles. Wiley-VCH 1983, Ch. 9-12. 
(9) de Abajo, F. J. G. Microscopy: Plasmons go quantum. Nature 2012, 483, 417-418.

(10) Scholl, J. A.; Koh, A. L.; Dionne, J. A. Quantum plasmon resonances of individual metallic nanoparticles. Nature 2012, 483, 421-427.

(11) Haberland, H. Looking from both sides. Nature 2013, 494, E1-E2.

(12) Malola, S.; Lehtovaara, L.; Enkovaara, J.; Häkkinen, H. Birth of the Localized Surface Plasmon Resonance in Monolayer-Protected Gold Nanoclusters. ACS Nano 2013, 7, $10263-10270$.

(13) Qian, H.; Zhu, Y.; Jin, R. Atomically precise gold nanocrystal molecules with surface plasmon resonance. Proceedings of the National Academy of Sciences 2012, 109, 696700 .

(14) Weissker, H.-C.; Escobar, H. B.; Thanthirige, V. D.; Kwak, K.; Lee, D.; Ramakrishna, G.; Whetten, R.; ; López-Lozano, X. Information on quantum states pervades the visible spectrum of the ubiquitous Au144 gold nanocluster. Nature Communications 2014, 5, 3785 .

(15) Desireddy, A.; Conn, B. E.; Guo, J.; Yoon, B.; Barnett, R. N.; Monahan, B. M.; Kirschbaum, K.; Griffith, W. P.; Whetten, R. L.; Landman, U. et al. Ultrastable silver nanoparticles. Nature 2013, 501, 399-402.

(16) Chakraborty, I.; Erusappan, J.; Govindarajan, A.; Sugi, K. S.; Udayabhaskararao, T.; Ghosh, A.; Pradeep, T. Emergence of metallicity in silver clusters in the 150 atom regime: a study of differently sized silver clusters. Nanoscale 2014, 6, 8024-8031.

(17) Alvarez, M. M.; Khoury, J. T.; Schaaff, T. G.; Shafigullin, M. N.; Vezmar, I.; Whetten, R. L. Optical Absorption Spectra of Nanocrystal Gold Molecules. The Journal of Physical Chemistry B 1997, 101, 3706-3712. 
(18) Chen, S.; Ingram, R. S.; Hostetler, M. J.; Pietron, J. J.; Murray, R. W.; Schaaff, T. G.; Khoury, J. T.; Alvarez, M. M.; Whetten, R. L. Gold Nanoelectrodes of Varied Size: Transition to Molecule-Like Charging. Science 1998, 280, 2098-2101.

(19) Schaaff, T. G.; Shafigullin, M. N.; Khoury, J. T.; Vezmar, I.; Whetten, R. L. Properties of a Ubiquitous $29 \mathrm{kDa}$ Au:SR Cluster Compound. The Journal of Physical Chemistry B 2001, 105, 8785-8796.

(20) Chaki, N. K.; Negishi, Y.; Tsunoyama, H.; Shichibu, Y.; Tsukuda, T. Ubiquitous 8 and 29 kDa Gold:Alkanethiolate Cluster Compounds: Mass-Spectrometric Determination of Molecular Formulas and Structural Implications. Journal of the American Chemical Society 2008, 130, 8608-8610, PMID: 18547044.

(21) Wong, O. A.; Heinecke, C. L.; Simone, A. R.; Whetten, R. L.; Ackerson, C. J. Ligand symmetry-equivalence on thiolate protected gold nanoclusters determined by NMR spectroscopy. Nanoscale 2012, 4, 4099-4102.

(22) Bahena, D.; Bhattarai, N.; Santiago, U.; Tlahuice, A.; Ponce, A.; Bach, S. B. H.; Yoon, B.; Whetten, R. L.; Landman, U.; Jose-Yacaman, M. STEM Electron Diffraction and High-Resolution Images Used in the Determination of the Crystal Structure of the $\mathrm{Au}_{144}(\mathrm{SR})_{60}$ Cluster. The Journal of Physical Chemistry Letters 2013, 4, 975-981.

(23) Yamazoe, S.; Takano, S.; Kurashige, W.; Yokoyama, T.; Nitta, K.; Negishi, Y.; Tsukuda, T. Hierarchy of bond stiffnesses within icosahedral-based gold clusters protected by thiolates. Nat. Commun. 7 .

(24) Polymorphism in magic-sized Au144(SR)60 clusters. Nat. Commun. 7.

(25) Weissker, H.-C.; Lopez-Acevedo, O.; Whetten, R. L.; López-Lozano, X. Optical Spectra of the Special Au144 Gold-Cluster Compounds: Sensitivity to Structure and Symmetry. The Journal of Physical Chemistry C 2015, 119, 11250-11259. 
(26) Fedrigo, S.; Harbich, W.; Buttet, J. Collective dipole oscillations in small silver clusters embedded in rare-gas matrices. Phys. Rev. B 1993, 47, 10706-10715.

(27) Cottancin, E.; Celep, G.; Lermé, J.; Pellarin, M.; Huntzinger, J.; Vialle, J.; Broyer, M. Optical Properties of Noble Metal Clusters as a Function of the Size: Comparison between Experiments and a Semi-Quantal Theory. Theoretical Chemistry Accounts: Theory, Computation, and Modeling (Theoretica Chimica Acta) 2006, 116, 514.

(28) López-Lozano, X.; Mottet, C.; Weissker, H.-C. Effect of Alloying on the Optical Properties of Ag/Au Nanoparticles. The Journal of Physical Chemistry C 2013, 117, 30623068.

(29) Kumara, C.; Dass, A. $(\mathrm{AuAg})_{144}(\mathrm{SR})_{60}$ alloy nanomolecules. Nanoscale 2011, 3, 30643067.

(30) Barcaro, G.; Sementa, L.; Fortunelli, A.; Stener, M. Comment on "(Au-Ag)144(SR)60 alloy nanomolecules" by C. Kumara and A. Dass, Nanoscale, 2011, 3, 3064. Nanoscale $2014,-$.

(31) Weissker, H.-C.; Mottet, C. Optical properties of pure and core-shell noble-metal nanoclusters from TDDFT: The influence of the atomic structure. Phys. Rev. B 2011, 84, 165443.

(32) Dharmaratne, A. C.; Dass, A. $\mathrm{Au}_{144-x} \mathrm{Cu}_{x}\left(\mathrm{SC}_{6} \mathrm{H}_{13}\right)_{60}$ nanomolecules: effect of $\mathrm{Cu}$ incorporation on composition and plasmon-like peak emergence in optical spectra. Chem. Commun. 2014, 50, 1722-1724.

(33) Bhattarai, N.; Black, D. M.; Boppidi, S.; Khanal, S.; Bahena, D.; Tlahuice-Flores, A.; Bach, S. B. H.; Whetten, R. L.; Jose-Yacaman, M. ESI-MS Identification of Abundant Copper-Gold Clusters Exhibiting High Plasmonic Character. The Journal of Physical Chemistry $C$ 2015, 119, 10935-10942. 
(34) Malola, S.; Hartmann, M. J.; Häkkinen, H. Copper Induces a Core Plasmon in Intermetallic $\mathrm{Au}(144,145)-\mathrm{xCux}(\mathrm{SR}) 60$ Nanoclusters. The Journal of Physical Chemistry Letters 2015, 6, 515-520.

(35) Yabana, K.; Bertsch, G. F. Time-dependent local-density approximation in real time. Phys. Rev. B 1996, 54, 4484-4487.

(36) Marques, M. A. L.; Castro, A.; Bertsch, G. F.; Rubio, A. Comp. Phys. Comm. 2003, 151,60 .

(37) Casida, M. E. Recent Advances in Density Functional Methods, Part I. 1995; p 155.

(38) Casida, M. E. Recent Developments and Applications of Modern Density Functional Theory. 1996; p 391.

(39) Lopez-Lozano, X.; Barron, H.; Mottet, C.; Weissker, H.-C. Aspect-ratio- and sizedependent emergence of the surface-plasmon resonance in gold nanorods - an ab initio TDDFT study. Phys. Chem. Chem. Phys. 2014, 16, 1820-1823.

(40) Lopez-Acevedo, O.; Akola, J.; Whetten, R. L.; Grönbeck, H.; Häkkinen, H. Structure and Bonding in the Ubiquitous Icosahedral Metallic Gold Cluster $\operatorname{Au}_{144}(\mathrm{SR})_{60}$. The Journal of Physical Chemistry C 2009, 113, 5035-5038.

(41) Pines, D. Elementary excitations in solids; Benjamin: New-York, 1963.

(42) Bernadotte, S.; Evers, F.; Jacob, C. R. Plasmons in molecules. The Journal of Physical Chemistry C 2013, 117, 1863-1878.

(43) Yannouleas, C.; Vigezzi, E.; Broglia, R. A. Evolution of the optical properties of alkalimetal microclusters towards the bulk: The matrix random-phase-approximation description. Phys. Rev. B 1993, 47, 9849-9861. 
(44) Weissker, H.-C.; Lopez-Lozano, X. Surface plasmons in quantum-sized noble-metal clusters: TDDFT quantum calculations and the classical picture of charge oscillations. Phys. Chem. Chem. Phys. 2015, 17, 28379-28386.

(45) Kresse, G.; Hafner, J. Ab initio molecular dynamics for liquid metals. Phys. Rev. B 1993, $47,558$.

(46) Kresse, G.; Furthmüller, J. Efficiency of ab-initio total energy calculations for metals and semiconductors using a plane-wave basis set. Comput. Mat. Sci. 1996, 6, 15.

(47) Kresse, G.; Joubert, D. From ultrasoft pseudopotentials to the projector augmentedwave method. Phys. Rev. B 1999, 59, 1758.

(48) Castro, A.; Marques, M. A. L.; Appel, H.; Oliveira, M.; Rozzi, C.; Andrade, X.; Lorenzen, F.; Gross, E. K. U.; Rubio, A. Phys. Stat. Sol. (b) 2006, 243, 2465.

(49) Perdew, J. P.; Burke, K.; Ernzerhof, M. Generalized Gradient Approximation made simple. Phys. Rev. Lett. 1996, 77, 3865.

(50) Troullier, N.; Martins, J. L. Efficient pseudopotentials for plane-wave calculations. Phys. Rev. B 1991, 43, 1993-2006. 
Graphical TOC Entry

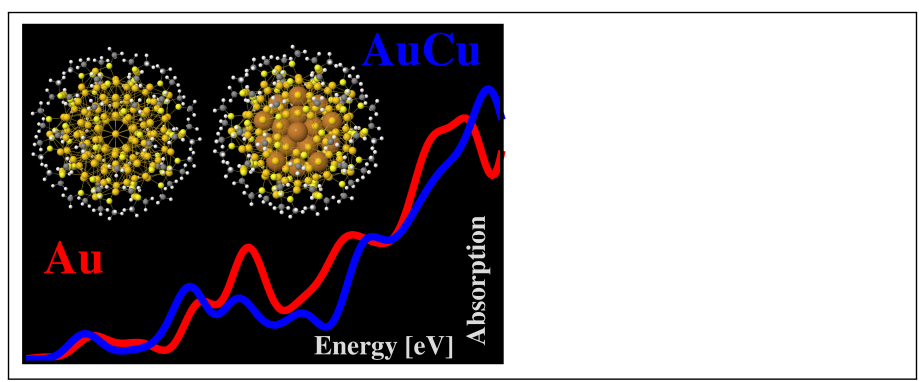

